\title{
High-resolution genomic profiling reveals gain of chromosome 14 as a predictor of poor outcome in ileal carcinoids
}

\author{
Ellinor Andersson ${ }^{1}$, Christina Swärd ${ }^{2}$, Göran Stenman ${ }^{1}$, Håkan Ahlman $^{2}$ \\ and Ola Nilsson ${ }^{1}$
}

${ }^{1}$ Lundberg Laboratory for Cancer Research, Department of Pathology and ${ }^{2}$ Lundberg Laboratory for Cancer Research, Department of Surgery, Sahlgrenska University Hospital, Gula Stråket 8, SE-413 45 Göteborg, Sweden

(Correspondence should be addressed to O Nilsson; Email: ola.nilsson@llcr.med.gu.se)

\begin{abstract}
Ileal carcinoids are malignant neuroendocrine tumours of the small intestine. The aim of this study was to obtain a high-resolution genomic profile of ileal carcinoids in order to define genetic changes important for tumour initiation, progression and survival. Forty-three patients with ileal carcinoids were investigated by high-resolution array-based comparative genomic hybridization. The average number of copy number alterations (CNAs) per tumour was 7.1 (range 1-22), with losses being more common than gains (ratio 1.4). The most frequent CNA was loss of chromosome $18(74 \%)$. Other frequent CNAs were gain of chromosome 4, 5, 14 and 20, and loss of 11q22.1-q22.2, 11q22.3-q23.1 and 11q23.3, and loss of 16q12.2-q22.1 and 16q23.2-qter. Two distinct patterns of CNAs were found; the majority of tumours was characterized by loss of chromosome 18 while a subgroup of tumours had intact chromosome 18, but gain of chromosome 14. Survival analysis, using a series of Poisson regressions including recurrent CNAs, demonstrated that gain of chromosome 14 was a strong predictor of poor survival. In conclusion, high-resolution profiling demonstrated two separate patterns of CNAs in ileal carcinoids. The majority of tumours showed loss of chromosome 18, which most likely represents a primary event in the development and pathogenesis of tumours. A different genetic pathway is operative in a subgroup of tumours; this is characterized by gain of chromosome 14 and is strongly associated with poor prognosis. Predictive fluorescence in situ hybridization analysis of chromosome 14 status in patients with ileal carcinoids is suggested.
\end{abstract}

Endocrine-Related Cancer (2009) 16 953-966

\section{Introduction}

Ileal carcinoids are malignant neuroendocrine tumours of the small intestine. The annual incidence of ileal carcinoids is 0.31 per 100000 and it is increasing (Modlin et al. 2003). They are slow-growing tumours and usually present late with either local manifestations (mass, bleeding and bowel obstruction) or symptoms due to overproduction of hormones (the midgut carcinoid syndrome). The overall 5-year survival rate for ileal carcinoids in the SEERs database is $64 \%$, with a significant difference in survival for localized and distant disease (73.8 and 46.4\% respectively; Modlin et al. 2003). Significantly better 5 -year survival rates have been reported from single centers after active interventions (Wängberg et al. 1996, Sarmiento \& Que 2003). The only curative treatment is radical surgery, but for palliation of symptoms the treatment can include cytoreductive surgery, ischaemic treatment of liver metastases or medical therapy with somatostatin analogues sometimes combined with interferon $\alpha$ (Kvols et al. 1986, Faiss et al. 2003, Kölby et al. 2003, Roche et al. 2003, Osborne et al. 2006). Recently, somatostatin receptormediated radiotherapy has been introduced as palliative treatment (Kwekkeboom et al. 2005, 2008). Ileal carcinoids are composed of enterochromaffin cells that secrete serotonin and tachykinins, which may give rise to hormonal symptoms. Other tumour markers are 
chromogranin A and vesicular monoamine transporter 1 (Stridsberg et al. 1995, Jakobsen et al. 2001). The aetiology of ileal carcinoids is unknown. The majority of tumours occurs sporadically and familial cases are extremely rare. Our knowledge about the genetic and epigenetic alterations that lead to ileal carcinoid formation is limited and pathogenetic mechanisms have not yet been identified. Several genes have been studied, but no mutations have been identified in TP53, KRAS, CTNNB1, CDKN2A, SMAD2, SMAD4 and BRAF (Weckström et al. 1996, Younes et al. 1997, Löllgen et al. 2001, Chan et al. 2003, Wang et al. 2005, Kulke et al. 2008). However, epigenetic changes such as genome-wide hypomethylation of LINE-1 and Alu-repetitive sequences and promoter hypermethylation of specific genes, i.e. RASSF1A, CTNNB1, LAMB3, CDKN2A, CDH1, $L A M C 2$ and THBS1, have been reported in a subset of tumours (Chan et al. 2003, Liu et al. 2005, Zhang et al. 2006, Choi et al. 2007).

There have been few cytogenetic studies of ileal carcinoids, mainly due to difficulties in obtaining goodquality metaphase chromosomes (Pfragner et al. 1996, Sjögren et al. 2000, Van Buren et al. 2007). Thus, little is known about structural alterations, e.g. chromosome translocations, in these tumours. Using microsatellite markers, chromosome-based comparative genomic hybridization (CGH) and single nucleotide polymorphism (SNP) analysis, recurrent copy number alterations (CNAs) have been identified. The most common CNA is loss of chromosome 18 (Terris et al. 1998, Zhao et al. 2000, Kytölä et al. 2001, Löllgen et al. 2001, Tönnies et al. 2001, Stancu et al. 2003, Wang et al. 2005, Kim do et al. 2008, Kulke et al. 2008). Other recurrent CNAs include losses involving chromosomes 9, 11q and/or 16q, and gains involving chromosomes 4, 5, 7, 14 and/or 20. No correlations have so far been identified between CNAs and clinicopathological data, most likely because ileal carcinoids are rare tumours and the fact that previous studies have been based on small numbers and used low-resolution techniques (Kim do et al. 2008, Kulke et al. 2008).

In order to obtain a high-resolution genomic profile of ileal carcinoids, we performed array-based CGH (aCGH). Samples from a total of 43 patients were analyzed and the results were correlated to clinical and histopathological features. We found multiple and recurrent CNAs in ileal carcinoids, the most frequent of which was loss of chromosome 18. In addition, we found a strong correlation between gain of chromosome 14 and poor survival.

\section{Material and methods}

\section{Tumour material}

Forty-three patients who underwent surgery for ileal carcinoids at the Sahlgrenska University Hospital, Göteborg, Sweden, from 1991 to 2007 were included. Fresh samples were collected at the time of surgery. Samples from a total of 24 primary tumours and 28 liver metastases were obtained; in nine cases, both primary tumour and liver metastases were available from the same patient. We retrieved complete clinical data for all patients including age at diagnosis, sex, hormonal symptoms, carcinoid heart disease (defined as tricuspid regurgitation by echocardiography), surgery, somatostatin analogues, hepatic arterial embolization and survival (Westberg et al. 2001). The histopathological evaluation was performed on haematoxylin-eosinstained sections. The immunohistochemical analysis included staining for chromogranin A, serotonin, synaptophysin, cytokeratin 8/18 and Ki67. Diagnostic criteria for ileal carcinoids were according to the World Health Organization (WHO) classification (Capella et al. 2000). Grading and staging were according to ENETS recommendations (Rindi et al. 2007). Staging of tumours was performed by assessing tumour-nodemetastasis (TNM). Grading of tumours was based on mitotic count and Ki67 index. Grade 1 tumours (G1) had $<2$ mitoses/10HPF and $\leq 2 \% \mathrm{Ki} 67$, grade 2 tumours (G2) had 2-20 mitoses/10HPF and 3-20\% Ki67 and grade 3 tumours (G3) had $>20$ mitoses/10HPF and $>20 \% \mathrm{Ki} 67$. Samples used for aCGH analysis were verified by histological examination. Only samples with a purity of at least $70 \%$ tumour cells were included.

\section{aCGH analysis}

Oligonucleotide-based aCGH (G4410B/G4426A/ G4426B; Agilent Technologies, Palo Alto, CA, USA) containing $\sim 44000$ probes distributed over all chromosomes were used. Both coding and non-coding sequences were covered, with a median probe spatial resolution of $43 \mathrm{~kb}$. The probes were annotated against NCBI genome build 36 (UCSC hg18, March 2006) and all mapped genes were represented by at least one probe. High molecular weight genomic DNA was extracted from fresh-frozen tumour specimens using the QIAamp DNA Mini Kit (Qiagen, Hilden, Germany). Human genomic DNA (Promega, Madison, WI, USA) pooled from normal males and females was used as reference DNA. Labelling and hybridization of DNA were performed according to the manufacturer's protocol. Briefly, $1.5 \mu \mathrm{g}$ high-quality DNA was fragmented by restriction digestion with AluI and 
RsaI (Promega). Subsequently, the digested DNA was labelled using random primers, exo-Klenow fragment and fluorescence-labelled nucleotides (Genomic DNA Labelling Kit Plus; Agilent Technologies). Tumour DNA was labelled with Cyanine 5 and reference DNA with Cyanine 3 for $2 \mathrm{~h}$ at $37^{\circ} \mathrm{C}$. After termination of the reaction and clean-up using a Microcon YM-30 filter (Millipore, Billerica, MA, USA), the incorporation of dye was determined spectrophotometrically. Sex-matched tumour and reference DNAs for each hybridization were pooled and mixed with Cot-1 DNA (Invitrogen, Carlsbad, CA, USA), blocking agent and hybridization solution (Oligo aCGH Hybridization Kit; Agilent Technologies). For each hybridization mixture, $100 \mu \mathrm{l}$ were pipetted onto the array and a gasket slide was placed on top. Hybridization was carried out for $24 \mathrm{~h}$ at $65{ }^{\circ} \mathrm{C}$ in a rotation oven $(\mathrm{G} 2545 \mathrm{~A}$; Agilent Technologies). After hybridization, the arrays were washed in Oligo aCGH Wash Buffers (Agilent Technologies) and Stabilization and Drying Solution (Agilent Technologies). Arrays were scanned at $5 \mu \mathrm{m}$ resolution using the Agilent's Microarray Scanner G2565BA (Agilent Technologies), and images were read and processed using Feature Extraction version 9.1 (G2567AA; Agilent Technologies). Data were further analyzed using CGH Analytics version 3.4 software (G4175AA, Agilent Technologies) using the moving average (window size: 1.0) and $z$-score algorithm (threshold: 2.5 ). A $\log _{2}$ ratio of \pm 0.3 in $>5$ consecutive probes was considered as gain/loss. A $\log _{2}$ ratio of $>2$ was designated as high-level amplification and a $\log _{2}$ ratio of $<-2$ was designated as homozygous loss. CNAs identified by the software were confirmed by visual inspection. Recurrent CNAs were controlled for copy number variations using the Database of Genomic Variants (Iafrate et al. 2004).

\section{Fluorescence in situ hybridization analysis}

Fluorescence in situ hybridization (FISH) analysis was performed on paraffin sections using a telomerespecific probe for chromosome 14 (TelVysion 14q SpectrumOrange; Abbott Molecular Inc., Des Plaines, IL, USA) according to the manufacturer's protocol. Briefly, $1 \mu \mathrm{m}$ paraffin sections of formalin-fixed tumour specimens were deparaffinized and pretreated with $0.2 \mathrm{~mol} / \mathrm{l} \mathrm{HCl}$ at room temperature for $20 \mathrm{~min}$ and with $1 \mathrm{~mol} / \mathrm{l}$ sodium thiocyanate for $30 \mathrm{~min}$ at $80{ }^{\circ} \mathrm{C}$. Subsequently, the sections were treated with Protease II (Abbott Molecular Inc.) for $30 \mathrm{~min}$ at $37^{\circ} \mathrm{C}$, dehydrated in increasing concentrations of ethanol and air-dried. Probe solutions were then applied and coverslips were placed on top and sealed with rubber cement. Denaturation $\left(5 \mathrm{~min}\right.$ at $\left.73{ }^{\circ} \mathrm{C}\right)$ and hybridization $\left(20 \mathrm{~h}\right.$ at $\left.37^{\circ} \mathrm{C}\right)$ were carried out in a hybridization chamber (Hybridizer; Dako, Glostrup, Denmark). After washing with $2 \times$ SSC containing $0.3 \% \mathrm{v} / \mathrm{v} \mathrm{NP}-40$ for $10 \mathrm{~min}$ at $72{ }^{\circ} \mathrm{C}$, sections were counterstained with DAPI I (Abbott Molecular Inc). Fluorescence signals were analyzed using a Zeiss Axioplan 2 fluorescence microscope (Carl Zeiss MicroImaging, Inc., Thornwood, NY, USA). The numbers of fluorescence signals were evaluated in at least 300 tumour cell nuclei from each of the specimens.

\section{Survival analysis}

The overall survival of patients with ileal carcinoids was analyzed using a series of Poisson regressions, including time since diagnosis, age, sex and recurrent CNAs included one by one. A patient was considered to be positive for a recurrent $\mathrm{CNA}$ if the CNA was demonstrated in the primary tumour and/or the liver metastasis. This analysis generated significant variables that were further evaluated in a stepwise multivariate analysis. Hormonal symptoms, carcinoid heart disease and Ki67 were included in the multivariate analysis. $P$ values $<0.05$ were considered significant. The programmes used for estimating death hazard functions by Poisson regression and for the calculation of Kaplan-Meier curves were developed by the statistical consultant (Prof. Anders Odén).

\section{Ethics}

This study was approved by the Regional Ethical Review Board in Göteborg, Sweden.

\section{Results}

The clinical and histopathological data are summarized in Table 1. All cases involved malignant ileal carcinoids composed of enterochromaffin cells immunoreactive against serotonin and chromogranin A. All tumours were we 11-differentiated endocrine carcinomas; the majority was of G1 (37 out of 43) and few were of G2 (6 out of 43). Most cases involved distant disease (stage IV; 38 out of 43), but a minority involved regional disease (stage IIIB; 4 out of 43 ) and there was a single case of localized disease (stage IIB; 1 out of 43).

\section{Ileal carcinoids carry multiple and recurrent CNAs}

CNAs were identified in all primary tumours and liver metastases; these are presented in Fig. 1 and Table 2. Altogether, 370 CNAs were detected in 52 tumour 
Primary tumour

\begin{tabular}{|c|c|c|c|c|c|c|c|c|c|c|c|c|c|c|c|c|c|c|}
\hline \multirow{2}{*}{$\frac{\text { Case }}{1}$} & \multirow{2}{*}{$\begin{array}{c}\begin{array}{c}\text { Age/ } \\
\text { sex }\end{array} \\
59 / M\end{array}$} & \multirow{2}{*}{$\begin{array}{c}\begin{array}{c}\text { Size } \\
(\mathrm{mm})\end{array} \\
10\end{array}$} & \multirow{2}{*}{ No. } & \multirow{2}{*}{$\begin{array}{c}\begin{array}{c}\text { Mitotic- } \\
\text { count }^{\mathrm{a}} \\
(10 \mathrm{HPF})\end{array} \\
<2\end{array}$} & \multirow{2}{*}{$\begin{array}{c}\mathrm{Ki67}^{\mathrm{a}} \\
(\%)\end{array}$} & \multicolumn{3}{|c|}{ TNM, grade and stage ${ }^{a}$} & \multirow{2}{*}{$\begin{array}{c}\begin{array}{c}\text { Primary } \\
\text { surgery }^{b}\end{array} \\
+\end{array}$} & \multirow{2}{*}{$\begin{array}{c}\text { Residual } \\
\text { disease } \\
\text { after } \\
\text { primary } \\
\text { surgery }\end{array}$} & \multirow{2}{*}{$\begin{array}{c}\text { HAE } \\
-\end{array}$} & \multirow{2}{*}{$\begin{array}{c}\begin{array}{c}\text { SSTR } \\
\text { analogs }\end{array} \\
-\end{array}$} & \multirow{2}{*}{$\frac{\text { IFN- } \alpha}{-}$} & \multirow{2}{*}{$\begin{array}{c}\begin{array}{c}\text { Carcinoid } \\
\text { heart } \\
\text { disease }^{\mathrm{c}}\end{array} \\
-\end{array}$} & \multirow{2}{*}{$\begin{array}{c}\text { Hormonal } \\
\text { symptom }\end{array}$} & \multirow{2}{*}{$\begin{array}{c}\begin{array}{c}\text { Other } \\
\text { malignancies }\end{array} \\
-\end{array}$} & \multirow{2}{*}{$\begin{array}{c}\begin{array}{c}\text { Follow-up } \\
\text { (mo.) }\end{array} \\
26\end{array}$} & \multirow{2}{*}{$\begin{array}{c}\begin{array}{c}\text { Current } \\
\text { status }\end{array} \\
\text { NED }\end{array}$} \\
\hline & & & & & & T3(m)N1M0 & G1 & IIIB & & & & & & & & & & \\
\hline 2 & $58 / F$ & 20 & 1 & $<2$ & $\leq 2$ & T3N1M1 & G1 & IV & $+^{d}$ & - & - & + & + & - & - & - & 88 & $A W D^{e}$ \\
\hline 3 & 69/F & 15 & 4 & $2-20$ & $\leq 2$ & T3(m)N1M0 & G2 & IIIB & + & $\mathrm{N}$ & - & + & + & - & + & - & 126 & AWD \\
\hline 4 & $71 / \mathrm{F}$ & 10 & 1 & $<2$ & $\leq 2$ & T3N1M1 & G1 & IV & + & L & 2 & + & - & + & + & - & 54 & DWD \\
\hline 5 & $53 / F$ & 17 & 2 & $<2$ & $\leq 2$ & T3(m)N1M1 & G1 & IV & + & $\mathrm{L}$ & - & + & - & - & + & - & 97 & AWD \\
\hline 6 & $74 / F$ & 25 & 2 & $<2$ & $\leq 2$ & $\mathrm{~T} 3(\mathrm{~m}) \mathrm{N} 1 \mathrm{M} 1$ & G1 & IV & $+^{f}$ & L, PE & 1 & + & - & - & + & $+^{9}$ & 83 & DWD \\
\hline 7 & $63 / F$ & 13 & 1 & $<2$ & $\leq 2$ & T3N1M1 & G1 & IV & + & $\mathrm{L}$ & 2 & + & + & + & + & - & 82 & DWD \\
\hline 8 & $66 / F$ & 13 & 1 & $<2$ & $\leq 2$ & T3N1M1 & G1 & IV & + & L & 2 & + & - & + & + & - & 57 & DWD \\
\hline 9 & $63 / \mathrm{M}$ & 20 & 5 & $<2$ & $\leq 2$ & T3(m)N1M1 & G1 & IV & $+^{d}$ & PE & - & + & + & - & + & - & 79 & AWD \\
\hline 10 & $71 / \mathrm{F}$ & 28 & 2 & $<2$ & $\leq 2$ & T3(m)NOMO & G1 & IIB & + & - & - & - & - & - & - & - & 78 & $\mathrm{AWD}^{\mathrm{e}}$ \\
\hline 11 & $49 / \mathrm{M}$ & 10 & 15 & $<2$ & $\leq 2$ & T3(m)N1M1 & G1 & IV & $+^{f}$ & L & - & + & + & - & - & - & 5 & DWD \\
\hline 12 & $68 / \mathrm{M}$ & 30 & 1 & $<2$ & $\leq 2$ & T3N1M1 & G1 & IV & + & $\mathrm{N}, \mathrm{L}$ & - & - & - & - & - & - & 22 & DWD \\
\hline 13 & $64 / F$ & 30 & 1 & $<2$ & $\leq 2$ & T4N1M1 & G1 & IV & + & L & 2 & + & + & + & + & $+^{\mathrm{h}}$ & 53 & DWD \\
\hline 14 & $42 / F$ & 22 & 1 & $<2$ & $\leq 2$ & T3N1M1 & G1 & IV & + & $\mathrm{L}$ & 5 & + & + & - & + & - & 120 & AWD \\
\hline 15 & $81 / \mathrm{F}$ & 30 & 1 & $<2$ & $\leq 2$ & T3N1M1 & G1 & IV & + & $\mathrm{L}, \mathrm{PE}$ & - & + & - & - & + & - & 35 & DWD \\
\hline 16 & $59 / \mathrm{M}$ & 3 & 1 & $<2$ & $\leq 2$ & T1N1M1 & G1 & IV & $t^{i}$ & $\mathrm{~L}$ & 2 & - & - & - & + & - & 57 & AWD \\
\hline 17 & $53 / \mathrm{F}$ & 25 & 2 & $<2$ & $\leq 2$ & T3(m)N1M1 & G1 & IV & + & $\mathrm{N}, \mathrm{L}, \mathrm{S}$ & - & + & + & - & + & - & 80 & DWD \\
\hline 18 & $68 / \mathrm{M}$ & 60 & 1 & $<2$ & $\leq 2$ & T3N1M1 & G1 & IV & + & L, PE & 2 & + & - & - & + & - & 18 & DWD \\
\hline 19 & $71 / \mathrm{F}$ & 20 & 1 & $<2$ & $\leq 2$ & T3N1M1 & G1 & IV & $+^{\mathrm{j}}$ & $\mathrm{L}, \mathrm{PE}$ & 4 & + & - & - & + & - & 33 & AWD \\
\hline 20 & 73/M & 5 & 1 & $<2$ & $\leq 2$ & T2N1M1 & G1 & IV & + & $\mathrm{L}$ & - & + & - & + & + & - & 6 & DWD \\
\hline 21 & $56 / F$ & 28 & 1 & $<2$ & $\leq 2$ & T3N1M1 & G1 & IV & $++^{k}$ & L & - & + & - & - & + & $+^{g}$ & 14 & DWD \\
\hline 22 & $53 / \mathrm{M}$ & 5 & 1 & $<2$ & $\leq 2$ & T3N1M1 & G1 & IV & + & L & 2 & + & + & - & + & - & 63 & DWD \\
\hline 23 & $80 / F$ & 60 & 1 & $<2$ & $\leq 2$ & T3N1M1 & G1 & IV & + & L & 2 & + & - & - & + & - & 116 & AWD \\
\hline 24 & $79 / \mathrm{M}$ & 12 & 1 & $<2$ & $\leq 2$ & T3N1M1 & G1 & IV & $+^{d}$ & $\mathrm{~N}, \mathrm{~L}, \mathrm{PE}$ & - & + & + & - & + & - & 24 & AWD \\
\hline 25 & $71 / \mathrm{M}$ & 6 & 1 & $<2$ & $\leq 2$ & T3N1M1 & G1 & IV & + & L & - & + & - & - & - & - & 111 & AWD \\
\hline 26 & $56 / F$ & 38 & 1 & $2-20$ & $3-20$ & T3N1M1 & G2 & IV & $+^{\prime}$ & $\mathrm{L}, \mathrm{PE}$ & 2 & + & + & - & + & - & 38 & AWD \\
\hline 27 & 70/M & 50 & 1 & $<2$ & $\leq 2$ & T3N1M1 & G1 & IV & + & $\mathrm{N}, \mathrm{L}$ & 1 & + & - & - & - & - & 15 & AWD \\
\hline 28 & $72 / M$ & 11 & 1 & $<2$ & $\leq 2$ & T3N1M1 & G1 & IV & + & $\mathrm{N}$ & - & + & - & - & + & - & 57 & AWD \\
\hline 29 & $64 / \mathrm{M}$ & 25 & 2 & $<2$ & $\leq 2$ & T3(m)N1M1 & G1 & IV & + & $\mathrm{N}, \mathrm{L}, \mathrm{S}$ & 3 & + & + & - & + & - & 97 & DWD \\
\hline 30 & $64 / F$ & 30 & 1 & $<2$ & $\leq 2$ & T3N1M1 & G1 & IV & $+^{\mathrm{d}, \mathrm{k}}$ & - & - & + & - & + & + & $+^{g}$ & 34 & NED \\
\hline 31 & $61 / \mathrm{F}$ & 25 & 1 & $2-20$ & $3-20$ & T3N1M1 & G2 & IV & + & $\mathrm{N}, \mathrm{PE}$ & - & + & + & - & + & $++^{g}$ & 15 & DWD \\
\hline 32 & $71 / \mathrm{M}$ & 9 & 5 & $<2$ & $\leq 2$ & T3(m)N1M1 & G1 & IV & $++^{k}$ & - & - & - & - & - & - & - & 80 & $A W D^{e}$ \\
\hline 33 & $61 / \mathrm{F}$ & 9 & 6 & $<2$ & $\leq 2$ & T3(m)N1M1 & G1 & IV & + & L & 2 & + & - & - & - & $+^{m}$ & 18 & AWD \\
\hline 34 & $70 / F$ & 25 & 1 & $2-20$ & $3-20$ & T3N1M1 & G2 & IV & $+^{i}$ & $\mathrm{~L}, \mathrm{PE}$ & 2 & + & - & - & - & - & 14 & DWD \\
\hline 35 & $73 / F$ & 20 & 1 & $2-20$ & $3-20$ & T3N1M1 & G2 & IV & + & $\mathrm{L}$ & 2 & - & - & - & + & - & 21 & DWD \\
\hline 36 & $75 / F$ & 14 & 2 & $<2$ & $\leq 2$ & T3(m)N1M1 & G1 & IV & $+^{f}$ & $\mathrm{~N}, \mathrm{~L}$ & 2 & + & + & - & + & - & 34 & DWD \\
\hline 37 & $60 / M$ & 27 & 1 & $<2$ & $\leq 2$ & T3N1M1 & G1 & IV & $+^{\mathrm{f}, \mathrm{k}}$ & - & - & - & - & - & + & - & 49 & $A W D^{e}$ \\
\hline 38 & $51 / \mathrm{M}$ & 50 & 1 & $2-20$ & $\leq 2$ & T3NOM1 & G2 & IV & + & $\mathrm{N}, \mathrm{L}$ & - & + & - & - & - & - & 54 & AWD \\
\hline 39 & $47 / \mathrm{F}$ & 6 & 1 & $<2$ & $\leq 2$ & T1N1M0 & G1 & IIIB & + & $\mathrm{N}$ & 2 & + & + & - & + & $+^{n}$ & 258 & AWD \\
\hline
\end{tabular}




\begin{tabular}{|c|c|c|c|c|c|c|c|c|c|c|c|c|c|c|c|c|c|c|}
\hline \multirow[b]{2}{*}{ Case } & \multirow[b]{2}{*}{$\begin{array}{c}\text { Age/ } \\
\text { sex }\end{array}$} & \multicolumn{4}{|c|}{ Primary tumour } & & & & \multirow[b]{2}{*}{$\begin{array}{l}\text { Primary } \\
\text { surgery }^{b}\end{array}$} & \multirow{2}{*}{$\begin{array}{c}\text { Residual } \\
\text { disease } \\
\text { after } \\
\text { primary } \\
\text { surgery }\end{array}$} & \multirow[b]{2}{*}{ HAE } & \multirow[b]{2}{*}{$\begin{array}{c}\text { SSTR } \\
\text { analogs }\end{array}$} & \multirow[b]{2}{*}{ IFN- $\alpha$} & \multirow[b]{2}{*}{$\begin{array}{c}\text { Carcinoid } \\
\text { heart } \\
\text { disease }^{c}\end{array}$} & \multirow[b]{2}{*}{$\begin{array}{l}\text { Hormonal } \\
\text { symptom }\end{array}$} & \multirow[b]{2}{*}{$\begin{array}{c}\text { Other } \\
\text { malignancies }\end{array}$} & \multirow[b]{2}{*}{$\begin{array}{l}\text { Follow-up } \\
\text { (mo.) }\end{array}$} & \multirow[b]{2}{*}{$\begin{array}{l}\text { Current } \\
\text { status }\end{array}$} \\
\hline & & $\begin{array}{l}\text { Size } \\
(\mathrm{mm})\end{array}$ & No. & $\begin{array}{l}\text { Mitotic- } \\
\text { count }^{\mathrm{a}} \\
(10 \mathrm{HPF})\end{array}$ & $\begin{array}{c}\mathrm{Ki67} \\
(\%)\end{array}$ & \multicolumn{3}{|c|}{ TNM, grade and stage ${ }^{a}$} & & & & & & & & & & \\
\hline 40 & $75 / F$ & 15 & 7 & $<2$ & $\leq 2$ & $\mathrm{~T} 3(\mathrm{~m}) \mathrm{N} 1 \mathrm{M} 1$ & G1 & IV & $t^{\mathrm{f}, \mathrm{k}}$ & L & 1 & + & + & - & + & - & 40 & DWD \\
\hline 41 & $53 / \mathrm{M}$ & 10 & 2 & $<2$ & $\leq 2$ & $\mathrm{~T} 3(\mathrm{~m}) \mathrm{N} 1 \mathrm{M} 0$ & G1 & IIIB & + & - & 4 & + & - & - & - & - & 110 & $A W D^{\mathrm{e}}$ \\
\hline 42 & $74 / \mathrm{M}$ & 25 & 1 & $<2$ & $\leq 2$ & T3N1M1 & G1 & IV & + & $\mathrm{N}, \mathrm{L}$ & - & + & + & - & + & $+^{\circ}$ & 16 & DWD \\
\hline 43 & 74/M & 15 & 1 & $<2$ & $\leq 2$ & T3N1M1 & G1 & IV & $+^{f}$ & $\mathrm{~N}, \mathrm{~L}$ & 2 & + & - & - & + & - & 38 & DWD \\
\hline
\end{tabular}

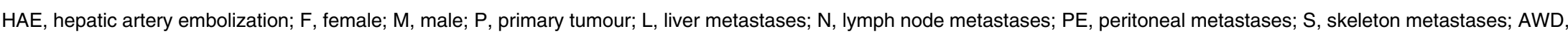
alive with disease; DWD, dead with disease; NED, no evidence of disease.

${ }^{a}$ According to ENETS proposal (see Ref. Rindi et al. (2007)).

${ }^{b}$ Includes resection of primary tumour, regional lymph nodes and cholecystectomy.

${ }^{\mathrm{C} D e f i n e d}$ as tricuspid regurgitation by echocardiography (see Ref. Westberg et al. (2001)).

dPrimary surgery and resection of peritoneal metastases.

Interval from primary surgery until recurrence: case 2 (42 mo.), case 10 (20 mo.), case 32 (11 mo.), case 37 (5 mo.) and case 41 (47 mo)

Primary surgery and high central lymph node resection.

${ }^{9}$ Breast cancer.

hMalignant melanoma.

'Primary surgery and para-aortal lymph node resection.

Primary surgery, sigmoideum and SOE bilateral resection.

${ }^{k}$ Primary surgery and liver resection.

'Primary surgery, hysterectomy and SOE resection.

mpapillary thyroid cancer.

${ }^{n}$ Gastrointestinal stromal tumour and colon cancer.

${ }^{\circ}$ Prostate cancer. 
Table 2 Copy number alterations identified by array-based comparative genomic hybridization in 43 patients with ileal carcinoid. Altogether, 52 samples were analyzed (24 primary tumours and 28 liver metastases)

\begin{tabular}{|c|c|c|c|}
\hline Case & $\mathrm{P} / \mathrm{M}$ & Gains & Losses \\
\hline 1 & $\mathrm{P}$ & & 18 \\
\hline 2 & $\mathrm{P}$ & & 18 \\
\hline 3 & $\mathrm{P}$ & & 18 \\
\hline 4 & $\mathrm{P}$ & & 18 \\
\hline 5 & $\mathrm{P}$ & & 18 \\
\hline 6 & $\mathrm{P}$ & & 18 \\
\hline 7 & $\mathrm{P}$ & 4 & 18 \\
\hline 8 & $\mathrm{P}$ & 20 & 18 \\
\hline 9 & $\mathrm{P}$ & & $16 p, 18$ \\
\hline 10 & $\mathrm{P}$ & $2 p 25.2-p 25.1$ & 18,22 \\
\hline 11 & $\mathrm{P}$ & 14 & $9 p, 3 p 21.31$ \\
\hline 12 & $\mathrm{P}$ & $17 q 25.1$ & 16q13-qter, 18 \\
\hline 13 & $\mathrm{P}$ & 5 pter-p11.21 & $11 \mathrm{q} 21-\mathrm{q} 24.2,16 \mathrm{q} 12.2-\mathrm{qter}, 18$ \\
\hline 14 & $\mathrm{P}$ & $\begin{array}{l}\text { 4pter-q13.13, 4q21.21-q21.23, 4q22.1-q22.3, } \\
4 q 25-q 28.3,4 q 31.22-q 32.2,5,20\end{array}$ & 1p35.1-p34.3, 1p32.2-p31.3, 1p31.2-p21.1, +1p13.2-p13.1, 16q, 18, X \\
\hline 15 & $\mathrm{P}$ & & $\begin{array}{l}\text { 1q32.1, 1q32.1, 1q32.1-q41, 3p25.1, 3p24.3-p24.2, 3p24.2, 3p24.1, 3p24.1-p22.2, 3p14.1, } \\
\text { 3p13, 4p13-q13.2, 9p11.1-q22.1, 11q13.5, 13q12.12-q12.2, 13q12.3, 13q14.2-q14.3, } \\
\text { 16q12.1-qter, } 18\end{array}$ \\
\hline \multirow[t]{2}{*}{16} & $\mathrm{P}$ & 20q11.23-qter & 18q22.2-qter \\
\hline & M & $12 q 14.1$ & $2 q 22.1-q 24.2,11 q 22.1-q 23.3$ \\
\hline \multirow[t]{2}{*}{17} & $\mathrm{P}$ & $6 q 27$ & 18 \\
\hline & M & $6 q 27$ & $11 q 14.2-q 24.1,15 q 26.1,17 q 25.3,18$ \\
\hline \multirow[t]{2}{*}{18} & $\mathrm{P}$ & & 18,22 \\
\hline & M & $4,5,7,8,10,14,15,17 \mathrm{p} 13.1$ & 11q14.1-qter, 13 \\
\hline \multirow[t]{2}{*}{19} & $P$ & $\begin{array}{l}3,4,5 q 14.1-q t e r, 14 q 11.2-q 13.1,14 q 23.3- \\
q 24.1,20\end{array}$ & \\
\hline & M & $3,4,5,8,12$ pter-p13.2, $14^{\mathrm{a}}, 20$ & \\
\hline \multirow[t]{2}{*}{20} & $P$ & $4,5,7,11 q 12.2,14^{a}, 16 p 11.2,20$ & 16q21-qter \\
\hline & M & $4 p, 5,7,14,16 q 11.2-q 12.1,20$ & 12p13.2-p11.22, 16q12.1-qter \\
\hline \multirow[t]{2}{*}{21} & $\mathrm{P}$ & $4,5,6,14,20$ & $3 p 21.31$ \\
\hline & $\mathrm{M}$ & $4,5,6,10 q 11.23-q 21.1,14^{a}, 20$ & $3 p 21.31,9,17 q 11.2,22$ \\
\hline \multirow[t]{2}{*}{22} & $P$ & & $2 q 22.3-q 24.3,7 p 22.2-p 12.1,18$ \\
\hline & M & $20 q$ & $\begin{array}{l}\text { 2q22.3-q24.3, 4p15.33, 7p22.1-p12.1, 9p22.2-p22.1, 9p21.3-p21.2, 9p21.1-p13.1, 9q, } \\
10 q 22.3,11 q 22.3-q 23.1,12 p 13.31-p 13.2,12 q 12,12 q 21.1,12 q 21.31,13 q 12.3,13 q 34,18\end{array}$ \\
\hline \multirow[t]{2}{*}{23} & $\mathrm{P}$ & $5 q 34.1,7 q 36.1,12 q 14.1$ & 18 \\
\hline & M & $\begin{array}{l}3 q 11.2-q 21.1,3 q 21.1-q 25.1,3 q 26.2-q 26.31 \\
\quad 4,5,21 q 22.13-q t e r\end{array}$ & $\begin{array}{l}\text { 2, 3p14.1-p12.3, 3p12.3-p12.1, 3q21.1, 3q25.1-q26.2, 3q26.31-q27.1, 3q29, 6p24.1-p22.3, 9, } \\
\text { 11, 13, 18, 21q22.13 }\end{array}$ \\
\hline
\end{tabular}


Table 2 continued

\begin{tabular}{|c|c|c|c|}
\hline Case & $\mathrm{P} / \mathrm{M}$ & Gains & Losses \\
\hline \multirow[t]{2}{*}{24} & $\mathrm{P}$ & $\begin{array}{l}4,5,12 p 12.3-p 11.22,12 q 21.2-q \text { ter, 14q24.3- } \\
\text { qter, 16q11.2-q12.2, } 20\end{array}$ & 12p13.2-p12.3, 12q12, 12q14.2-q21.1, 14q23.3, 14q24.1-q24.3, 16q12.2-qter, 18 \\
\hline & M & $\begin{array}{l}4,5,12 p 12.3-p 11.1,12 q 21.2-q t e r, 14 q 24.3- \\
\text { qter, 16q11.2-q12.2, } 20\end{array}$ & 12p13.2-p12.3, 12q12, 12q14.2-q21.1, 14q23.3, 14q24.1-q24.3, 16q12.2-qter, 18 \\
\hline 25 & M & & $16 q 12.1-q 22.1,18$ \\
\hline 26 & M & $20 p$ & $9 p, 18$ \\
\hline 27 & M & 4 & $1 \mathrm{p} 35.2-\mathrm{p} 35.1,18$ \\
\hline 28 & M & 20 & $3 p 14.1-p 12.3,3 q 22.1-q 22.3,13 q 14.2-q 14.3$ \\
\hline 29 & M & $7 \mathrm{p} 15.2$ & $11 q 14.1-q 24.2,12 p 13.31,17 p 13.1,18$ \\
\hline 30 & M & 3p21.31, 11q13.2, 12p13.31 & $18,22 q 11.21, X$ \\
\hline 31 & M & $4,5,7,10,14^{a}, 17 q 21.2-q t e r, 20$ & \\
\hline 32 & M & & $1 q 25.2-q 31.3,1 q 32.1-q 42.12,2 p 13.3,3 q 22.2-q 22.3,3 q 26.2-q 26.31,3 q 26.32-q 26.33,18$ \\
\hline 33 & M & 11q13.2, 17q12-qter, 21, X & $9,11 \mathrm{q} 14.1-\mathrm{q} 24.1,18$ \\
\hline 34 & M & $4,5,7,12 p 13.31,14^{a}, 20,21$ & $12 \mathrm{p} 13.31$ \\
\hline 35 & M & $1 \mathrm{q}, 4,5,7,10,12,14^{\mathrm{a}}, 20$ & $1 \mathrm{p}, 13$ \\
\hline 36 & M & 20 & $1 p, 8,9,13,15,17$ pter-q22, 18, 19, 21 \\
\hline 37 & M & & $\begin{array}{l}\text { 3p25.1-p24.2, 3p14.2-p12.3, 3q13.13-q13.2, 3q21.1-q21.2, 3q21.3-q22.1, 3q24-q26.1, } \\
\text { 3q26.2-q26.33, 8q12.1, 12p13.2-p13, } 18\end{array}$ \\
\hline 38 & M & & $\begin{array}{l}\text { 3p14.1-p12.3, 3q22.1, 5p15.2, 5q31.1, 5q31.3-q33.1, 5q33.2, 14q13.2, 14q13.3, 14q23.2- } \\
\text { q23.3, 14q24.1-q24.3, 18, 22q13.1-q13.2 }\end{array}$ \\
\hline 39 & M & $11 p 13,15 q 26.3$ & 2pter-p24.1, 3pter-q22.1, 6, 8p12, 9p, 11q14.1-qter, 12p13.2-p12.3, 13, 17pter-p13.1, 22 \\
\hline 40 & M & $1 \mathrm{q}, 4,20$ & $1 p, 2,3,6 q, 7 q 22.1,9,11 p 14.3-p 12,11 q 22.1-q 22.2,11 q 23.3-q 24.2,13,18$ \\
\hline 41 & M & $5,6,11,14^{a}, 17,20,21, x p 21.1$ & 3p26.2-p25.2, 3p14.1-p13, 3q13.13, 3q26.31-q29, 9, 12p13.31, 18 \\
\hline 42 & M & $4,5,10,14,20$ & $\begin{array}{l}\text { 1p36.32, 1p36.22, 1p36.21, 1p36.13-p36.12, 1p36.11-p35.2, 1p34.3-p31.3, 1p31.3-p31.1, } \\
\text { 1p31.1-p22.2, 2q21.1-q32.1, 13, 16q12.1 }\end{array}$ \\
\hline 43 & M & $\begin{array}{l}\text { 1p34.1-p33, 2q35, 3q27.3-q28, 4pter-q28.1, } \\
\text { 4q31.22-qter, 5q23.3, 6q12, 7p15.2-p14.3 } \\
\text { 7q21.11, 7q21.13-q21.3, 7q36.1, 11q22.1- } \\
\text { q22.2, 13q32.3-q33, 20, xp11.3-p11.22, } \\
\text { xp11.21-p11.1 }\end{array}$ & $1 p 22.3,4 q 31.21,13 q 13.1-q 22.3,16 q 23.2-q t e r, 18$ \\
\hline
\end{tabular}

$\mathrm{P}$, primary tumour; $\mathrm{M}$, liver metastasis.

${ }^{\mathrm{a} C}$ Confirmed by FISH analysis. 

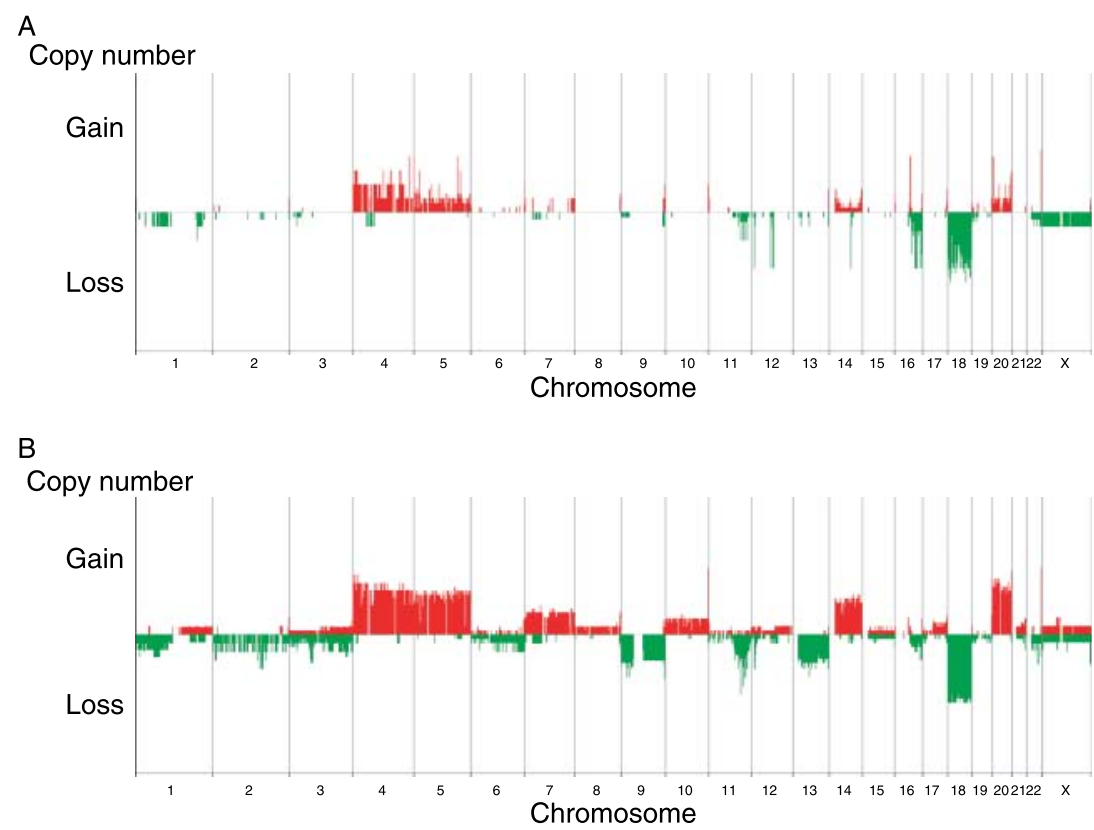

Figure 1 Genome-wide penetrance plot of CNAs in 24 primary tumours (A) and 28 liver metastases (B). Losses (green) were mainly detected on chromosome $9,11,13$ and 18, while gains (red) were mainly observed on chromosomes $4,5,14$ and 20 . Loss on chromosome 18 was the most frequent alteration, both in primary tumours and liver metastases.

samples. The average number of CNAs per tumour sample was 7.1 (range 1-22). Losses were more common than gains, with a ratio of 1.4. CNAs were detected most frequently on chromosome arms $18 \mathrm{q}$ $(71 \%), 18 \mathrm{p}(69 \%), 4 \mathrm{p}, 20 \mathrm{q}(40 \%$ each), 20p (38\%), 4q, $5 q(37 \%$ each), 14q (35\%), 5p (33\%), 11q (31\%), 12p (29\%), 3p and 16q (27\% each). Loss or gain of whole chromosomes accounted for $41 \%$ of all CNAs. No high-level amplifications were observed. Homozygous loss was observed in one patient. A comparison between primary tumours and liver metastases revealed that the average number of CNAs per tumour was twice as high in liver metastases (9.5, range 2-22) as in primary tumours (4.3, range 1-18). The proportions of losses and gains were similar in primary tumours and liver metastases.

Recurrent CNAs, defined as alterations occurring in three or more patients, accounted for $59 \%$ of all alterations. A summary of recurrent CNAs is given in Table 3. Detailed information on all recurrent CNAs is given in Supplementary Table 1, which can be viewed online at http://erc.endocrinology-journals.org/ supplemental/. aCGH profiles of individual tumours are given in Fig. 2. The most frequently recurring CNA was loss of an entire chromosome 18 , which was observed in $74 \%$ of all patients. Loss of chromosome 18 was detected with similar frequency in primary tumours (79\%) and liver metastases (61\%). Only one tumour (primary tumour) showed partial loss of 18q22.2-qter. Losses of chromosomes 9 and 13 were found at lower frequency (14 and $16 \%$ of patients respectively) and they were only observed in liver metastases.

The most frequently recurring segmental CNAs were found on chromosome 11, and included deletions of three minimal common regions (11q22.1-q22.2, 11q22.3-q23.1 and 11q23.3), all of which were present in $21 \%$ of the patients. Candidate tumour-suppressor genes in these regions include radixin $(R D X)$, B-cell translocation gene 4 (BTG4), protein phosphatase 2 , regulatory subunit $A$, beta isoform $(P P P 2 R 1 B)$ and transgelin $(T A G L N)$. Losses on chromosome 11 were three times more common in liver metastases than in primary tumours. Recurrent CNAs on chromosome 16 included two minimal common regions (16q12.2-q22.1 and 16q23.2-qter), which were lost in $16 \%$ of the patients. Candidate tumour-suppressor genes in these segments include $C D H$ 1, 3, 5, 8, 11, 13, 15, TRADD, E2F4, CTCF, OSGIN1, WFDC1, IRF8, FBXO31, BANP, CBFA2T3, ZNF276, FANCA and GAS8. Losses on chromosome 16 were twice as common in primary tumours as in liver metastases. Loss of 3p13 was observed in $14 \%$ of the patients, and it was four times as common in 
Table 3 Recurrent copy number alterations (CNAs) in 43 patients with ileal carcinoid analyzed by array-based comparative genomic hybridization

\begin{tabular}{|c|c|c|c|c|c|c|c|}
\hline \multirow[b]{2}{*}{ Chromosome } & \multirow{2}{*}{$\begin{array}{l}\text { Minimal } \\
\text { common } \\
\text { region }\end{array}$} & \multirow[b]{2}{*}{ Start and stop } & \multirow[b]{2}{*}{ Size (Mb) } & \multirow{2}{*}{$\begin{array}{c}\text { No. of } \\
\text { annotated } \\
\text { genes }^{a}\end{array}$} & \multicolumn{3}{|c|}{ Frequency of CNAs } \\
\hline & & & & & $\mathbf{P}^{\mathbf{b}}$ & $\mathbf{M}^{\mathbf{b}}$ & Patients $^{b}$ \\
\hline \multicolumn{8}{|l|}{ Losses } \\
\hline \multirow{2}{*}{1} & $1 p 22.3$ & $87123525-87715408$ & 0.6 & 2 & 1 & 2 & 3 \\
\hline & $1 p$ & & & & 0 & 3 & 3 \\
\hline 2 & $2 q 22.3-q 24.2$ & $147651413-162610374$ & 15 & 10 & 1 & 3 & 3 \\
\hline \multirow[t]{6}{*}{3} & $3 p 13$ & $72179608-73194811$ & 1.0 & 5 & 1 & 5 & 6 \\
\hline & $3 p 21.31$ & $48777730-48939935$ & 0.2 & 4 & 2 & 2 & 3 \\
\hline & $3 q 21.1$ & $124646570-124983177$ & 0.3 & 3 & 0 & 3 & 3 \\
\hline & $3 q 22.1$ & $132971150-133569797$ & 0.6 & 13 & 0 & 3 & 3 \\
\hline & $3 q 22.1$ & $134528771-135763122$ & 1.2 & 2 & 0 & 3 & 3 \\
\hline & $3 q 26.32-q 26.33$ & $178193148-180811471$ & 2.6 & 11 & 0 & 4 & 4 \\
\hline \multirow[t]{2}{*}{9} & $9 p$ & & & & 1 & 2 & 3 \\
\hline & 9 & & & & 0 & 6 & 6 \\
\hline \multirow[t]{3}{*}{11} & $11 q 22.1-q 22.2$ & 96830209-101803071 & 5.0 & 10 & 2 & 7 & 9 \\
\hline & $11 q 22.3-q 23.1$ & 108214469-111299004 & 3.1 & 20 & 2 & 7 & 9 \\
\hline & $11 \mathrm{q} 23.3$ & $115832077-118400328$ & 2.6 & 45 & 2 & 7 & 9 \\
\hline 12 & $12 \mathrm{p} 13.2$ & $11030657-12766204$ & 1.7 & 23 & 1 & 5 & 5 \\
\hline \multirow[t]{2}{*}{13} & $13 q 14.2-q 14.3$ & $48467994-51626277$ & 3.2 & 26 & 1 & 2 & 3 \\
\hline & 13 & & & & 0 & 7 & 7 \\
\hline \multirow[t]{3}{*}{16} & $16 q 12.1$ & $48563960-48816620$ & 0.3 & 3 & 1 & 2 & 3 \\
\hline & $16 q 12.2-q 22.1$ & $54446243-67347099$ & 13 & 133 & 6 & 3 & 7 \\
\hline & 16q23.2-qter & $78736208-88651780$ & 9.9 & 96 & 6 & 3 & 7 \\
\hline 18 & 18 & & & & 19 & 17 & 32 \\
\hline 22 & 22 & & & & 2 & 2 & 4 \\
\hline \multicolumn{8}{|l|}{ Gains } \\
\hline 4 & 4 & & & & 5 & 11 & 13 \\
\hline 5 & 5 & & & & 4 & 11 & 12 \\
\hline 7 & 7 & & & & 1 & 5 & 5 \\
\hline 10 & 10 & & & & 0 & 4 & 4 \\
\hline 14 & 14 & & & & 3 & 9 & 10 \\
\hline 20 & 20 & & & & 6 & 12 & 14 \\
\hline 21 & 21 & & & & 0 & 3 & 3 \\
\hline
\end{tabular}

${ }^{\mathrm{a} O n l y}$ the numbers of genes in defined chromosome segments are given.

${ }^{b}$ Primary tumours: $n=24$; liver metastases: $n=28$; patients: $n=43$.

liver metastases as in primary tumours. One candidate tumour-suppressor gene in this segment is $R Y B P$. There were no recurrent gains of specific chromosome segments. There were recurrent gains of whole chromosomes, however, most frequently on chromosomes $20(33 \%), 4(30 \%), 5(28 \%)$ and $14(23 \%)$. Gains of whole chromosomes were more frequent in metastatic tumours than in primary tumours.

\section{Two groups of ileal carcinoids with distinct patterns of CNAs}

Loss of chromosome 18 was the most common CNA in ileal carcinoids. Loss of an entire chromosome 18 was identified in 32 out of 43 patients $(74 \%)$, while 1 patient had partial loss of chromosome 18. Altogether, loss on chromosome 18 was demonstrated in 33 out of
43 patients $(77 \%)$. In six patients, loss of chromosome 18 was the only CNA observed (all in primary tumours). Apart from loss of chromosome 18, other CNAs were losses involving chromosomes 3, 9,11,13 and/or 16 and gains involving chromosomes 4, 5 and/or 20. In general, tumours with losses on chromosome 18 were characterized by a limited number of CNAs per tumour, with losses being more common than gains (ratio 2.1).

In ten patients, representing $23 \%$ of all cases, no CNA involving chromosome 18 was detected. This group of tumours was characterized by a limited number of CNAs per tumour, with losses being less common than gains (ratio 0.5). The most frequent gains in this group involved chromosomes 4, 5, 7, 10, 14 and 20 , and these occurred with similar frequency in primary tumours and liver metastases. 

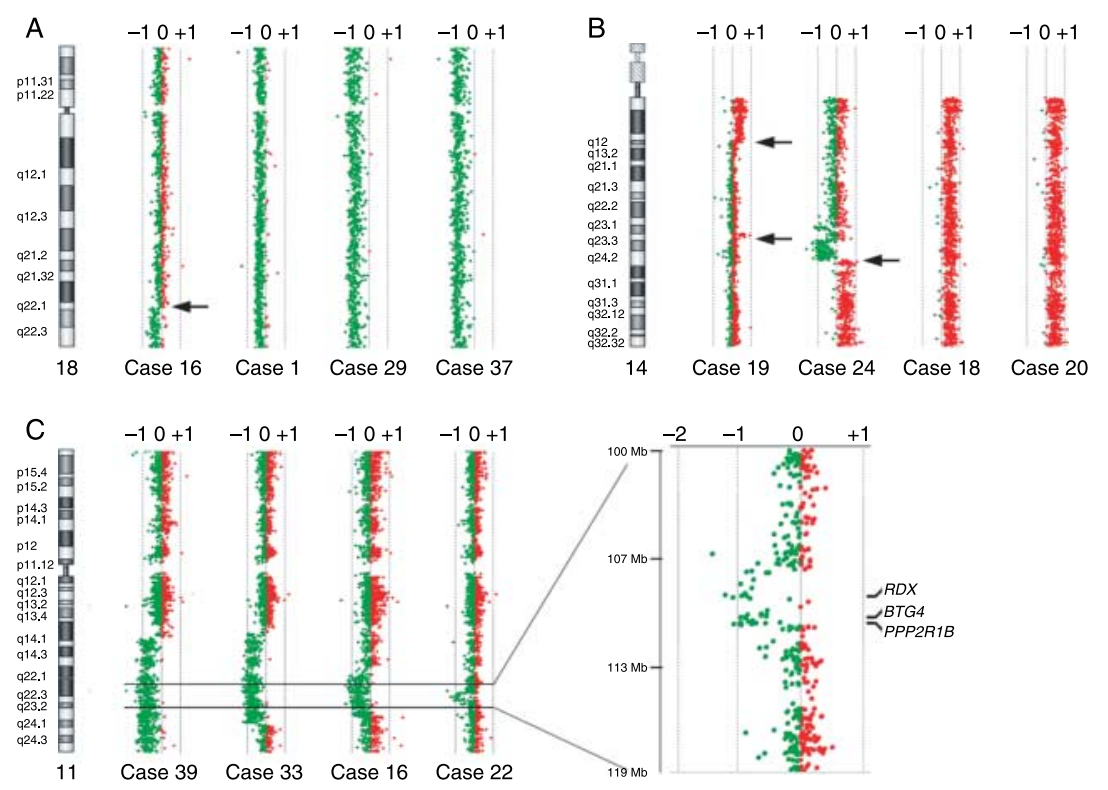

Figure 2 Examples of aCGH profiles illustrating recurrent CNAs in ileal carcinoids. (A) Profiles of chromosome 18 demonstrating segmental loss of 18q22.2-qter in one primary tumour (case 16) and loss of an entire chromosome 18 in one primary tumour (case 1) and two liver metastases (cases 29 and 37). (B) Profiles of chromosome 14 demonstrating segmental gains of 14q11.2-q13.1 (case 19, primary), 14q23.3-q24.1 (case 19, primary) and 14q24.3-qter (case 24, liver metastasis) and gain of an entire chromosome 14 in two liver metastases (cases 18 and 20). (C) Profiles of chromosome 11 demonstrating segmental losses with a minimal common region at 11q22.3-q23.1 (cases 39, 33, 16 and 22, liver metastases). The location of candidate tumour-suppressor genes $R D X$, BTG4 and PPP2R1B is indicated.

\section{Survival analysis suggested poor prognosis in patients with ileal carcinoids showing gain of chromosome 14}

Survival analysis was performed with respect to all recurrent CNAs detected in ileal carcinoids (Table 3). There was a significant correlation between four variables (gain of 7, 14 and 20, and loss of 18) and overall survival $(P<0.05)$; in this context, three variables (presence of gain of whole chromosomes in general, gain of chromosome 4 and loss of 3p13) were almost significant in the series of Poisson regressions $(P<0.1)$. Following multivariate analysis, there was a significant correlation between 1) loss of $3 p 13$ or 2 ) gain of chromosome 14 or 3) carcinoid heart disease and survival. Hormonal symptoms and Ki67 did not correlate with survival (see Supplementary Table 2, which can be viewed online at http://erc. endocrinology-journals.org/supplemental/). Patients with loss of $3 \mathrm{p} 13$ had a significantly reduced risk of death $(P=0.028)$ with a hazard ratio $(\mathrm{HR})$ of 0.14 (95\% CI: 0.02-0.8), while patients with gain of chromosome 14 had a significantly increased risk of death $(P<0.001)$ with an HR of 8.39 (95\% CI: 3.04-23.11). Patients with carcinoid heart disease also had a significantly increased risk of death $(P<0.044)$ with an HR of 2.98 (95\% CI: 1.03-8.63).
A Kaplan-Meier survival plot for patients with ileal carcinoids showing gain of chromosome 14 is shown in Fig. 3. There was gain of chromosome 14 in 10 out of 43 patients. Gain of chromosome 14 was more common in liver metastases ( 9 out of 28) than in primary tumours ( 3 out of 24 ). One patient with gain of

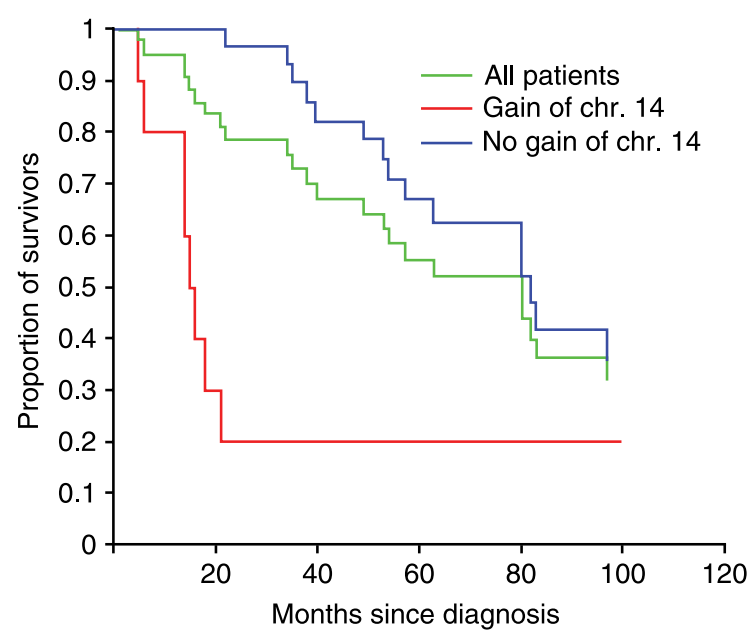

Figure 3 Kaplan-Meier survival analysis of ileal carcinoids. Patients with gain of chromosome 14 had a significantly worse prognosis than patients with no gain of chromosome 14 (HR 8.39, 95\% Cl: 3.04-23.11). 


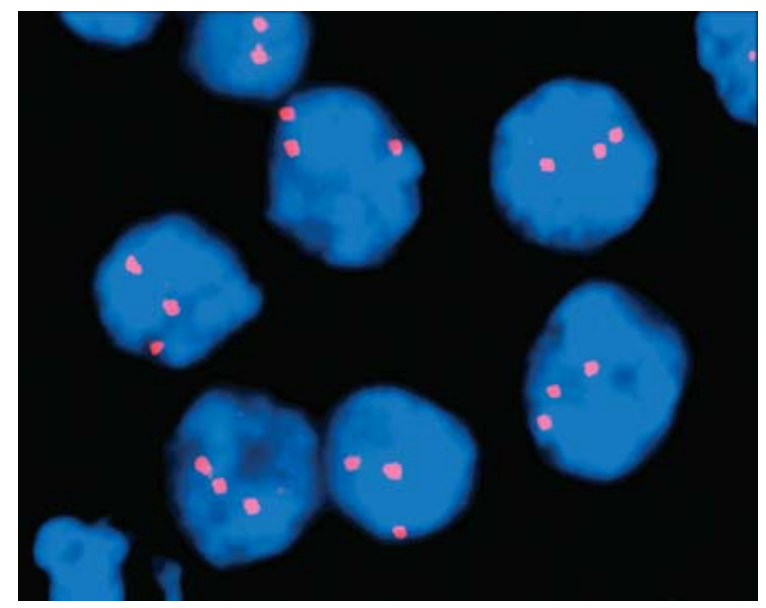

Figure $4 \mathrm{FISH}$ analysis of an ileal carcinoid with gain of chromosome 14. FISH analysis was performed on a paraffin section of a primary tumour (case 20), using a telomere-specific probe for chromosome 14, and confirmed the aCGH finding.

chromosome 14 had segmental gains in the primary tumour (14q11.2-q13.1 and 14q23.3-q24.1) and gain of whole chromosome 14 in the liver metastasis. Segmental gain of chromosome 14 (14q24.3-qter) was observed in another patient. Patients with gain of chromosome 14 rarely displayed loss of chromosome 18 (two cases only), but they frequently had gain of chromosomes 4, 5, 7, 10 and 20. Gain of chromosome 7 was found in five out of ten tumours with gain of chromosome 14, but it could not be identified in any of the tumours with loss of chromosome 18.

\section{FISH analysis of ileal carcinoids confirmed gain of chromosome 14}

Patients with gain of chromosome 14 identified in the aCGH analysis were analyzed by FISH using a telomeric probe for chromosome 14. The FISH analysis confirmed gain of chromosome 14 in seven out of seven patients analyzed (Fig. 4). Gain of chromosome 14 was not found in $100 \%$ of the tumour cells in any of the tumours. The frequency of trisomic cells varied from 36 to $86 \%$ in the different tumours.

\section{Discussion}

Here, we present for the first time an in-depth analysis of ileal carcinoids, involving high-resolution genomic profiling of tumours from a large number of patients with long follow-up (median 53 months, range 5-258 months). We identified loss of chromosome 18 as the most common CNA; it occurred in $74 \%$ of the patients with ileal carcinoids. Loss of chromosome 18 was detected with similar frequency in primary tumours and liver metastases, suggesting that changes involving chromosome 18 are early events in tumour formation. The question of which genes on chromosome 18 are important for carcinoid tumour formation could not be answered from our data. Only one of our patients displayed segmental loss on chromosome 18 (18q22.2-qter). This is in line with previous studies demonstrating losses of 18q12-qter, 18q21-qter, $18 \mathrm{q} 21.1$ or $18 \mathrm{q} 22$-qter in ileal carcinoids using chromosome-based CGH or SNP analysis (Zhao et al. 2000, Kytölä et al. 2001, Kulke et al. 2008). Candidate genes at $18 \mathrm{q} 21.1$ include SMAD2 and $S M A D 4$, but to date sequencing of these genes has not revealed any mutations (Löllgen et al. 2001, Kulke et al. 2008).

The most common segmental losses in ileal carcinoids occurred on 11q22.1-q22.2, 11q22.3-q23.1 and $11 \mathrm{q} 23.3$. These alterations were three times more frequent in liver metastases than in primary tumours, indicating that these changes are associated with tumour progression. Loss of 11q22-23 has also been described in a number of other solid tumours including carcinoma of the breast and colon, lymphoproliferative diseases and haematological malignancies. RDX, BTG4, PPP $2 R 1 B$ and TAGLN are candidate tumour-suppressor genes in these regions. $R D X$ is a member of the ezrin-radixin-moesin family of proteins, which act as cytoskeletal adopters and signalling proteins (Hoeflich \& Ikura 2004). Expression of $R D X$ can be downregulated in adenocarcinomas of the lung (Tokunou et al. 2000). BTG4 is a cell cycle regulator that induces G1 arrest. Loss of BTG4 is a common event in chronic lymphocytic leukaemia, and epigenetic silencing of $B T G 4$ by $\mathrm{CpG}$ island methylation occurs in colorectal cancer (Auer et al. 2005, Toyota et al. 2008). PPP2R1B regulates RaIA GTPase and inhibits cell growth (Sablina et al. 2007). Inactivation of $P P P 2 R 1 B$ by downregulation, or mutation, has been demonstrated in B-cell chronic lymphocytic leukaemia, lung cancer and colon cancer (Wang et al. 1998). TAGLN is an actin cross-linker protein involved in replicative senescence, and loss of this gene has also been demonstrated in breast and colon cancer (Shields et al. 2002, Yang et al. 2007). There are no reports of $R D X, B T G 4, P P P 2 R 1 B$ or TAGLN expression in ileal carcinoids, and the role of these tumour-suppressor genes in carcinoid tumour progression remains to be determined.

The second most common segmental loss occurred on chromosome 16, including the minimal common regions 16q12.2-q22.1 and 16q23.2-qter. Loss of these regions has been demonstrated in a large number of solid tumours - including tumours of the breast, 
prostate, liver, lung and ovary (Rakha et al. 2006). A large number of candidate tumour-suppressor genes are present in these regions, including a group of cadherins ( $C D H 1,3,5,8,11,13$ and 15). E-cadherin $(\mathrm{CDH1})$ is a key regulator of the epithelial-mesenchymal transition, which is activated during tumour invasion and dissemination (Schmalhofer et al. 2009). Downregulation of E-cadherin has been demonstrated in carcinoid tumours (Sun et al. 2002).

The third most common segmental loss occurred on 3 p13, which was four times more common in liver metastases than in primary tumours, indicating that this alteration accumulates during tumour progression. Only one tumour-suppressor gene, $R Y B P$, was present in this region. $R Y B P$ (RING1 and YY1 binding protein, also known as $D E D A F$ ) functions as a repressor of E4TF1 and induces apoptosis in tumour cells (Danen-van Oorschot et al. 2004). The preferential tumour-killing activity of RYBP has made it a promising target for cancer therapy (Novak \& Phillips 2008).

The pattern of CNAs in ileal carcinoids appears to be specific for this type of tumour and it is different from that previously reported for other well-differentiated neuroendocrine tumours, e.g. pancreatic endocrine tumours and lung carcinoids. In the latter tumours, losses on chromosome 11, including the MEN1 locus, have been shown to be a primary tumour oncogenic event (Walch et al. 1998, Zhao et al. 2001). When comparing carcinoid tumours with an intact chromosome 18 and tumours with loss of chromosome 18, two distinct patterns of CNAs appeared. Tumours with intact chromosome 18 had more gains of whole chromosomes, in particular gains of $4,5,7,10,14$ and 20, than tumours with loss of chromosome 18 . These findings suggest that carcinoid tumours may arise via two separate genetic pathways, as previously suggested by Kulke et al. (2008). Loss of chromosome 18 is of importance in the majority of tumours, while a different pathway may operate in a subset of tumours.

The perhaps most striking observation in the present series of carcinoids was that survival analysis demonstrated a strong correlation between gain of chromosome 14 and poor survival (HR 8.39; 95\% CI: 3.04-23.11). This is the first time that a genetic event has been linked to the clinical course of ileal carcinoids. We also confirmed our aCGH finding of gain of chromosome 14 by FISH analysis. In a series of 18 ileal carcinoids, Kulke et al. (2008) reported gain of $14 \mathrm{q} 11.2$ and suggested that $D A D 1$ might be a potential oncogene in the tumourigenesis. To identify candidate oncogenes on chromosome 14 that may be responsible for the poor prognosis is difficult in the present series of tumours, because segmental gains (i.e. $14 q 11.2-q 13.1,14 q 23.3-q 24.1$ and $14 q 24.3-q$ ter) were only observed in two patients. An interesting observation is that ileal carcinoids with gain of chromosome 14 usually had intact chromosome 18. However, gain of chromosome 14 and loss of chromosome 18 were not mutually exclusive, since two patients had both alterations. Gain of chromosome 14 was observed in both primary and metastatic tumours. We therefore suggest that carcinoid tumours with gain of chromosome 14 follow a different genetic pathway than the majority of ileal carcinoids. The clinical relevance of subgrouping carcinoid tumours according to CNAs should be evaluated in a larger series of patients. It is, however, clear that gain of chromosome 14 is an important indicator of poor prognosis. As a consequence, we suggest that biopsies from patients with malignant ileal carcinoids should be subjected to FISH analysis of chromosome 14, which can readily be performed on paraffin-embedded specimens. Patients with gain of chromosome 14 should be under strict surveillance in order to be able to treat recurrences at an early stage.

\section{Declaration of interest}

The authors declare that there is no conflict of interest that would prejudice the impartiality of this scientific work.

\section{Funding}

This work was supported by the Swedish Cancer Society; the Swedish Research Council; Sahlgrenska Academy (ALF grant); the Assar Gabrielsson Research Foundation; and the Sahlgrenska University Hospital Research Foundations.

\section{Acknowledgements}

We thank Anders Odén for statistical analysis and Malin Berntsson and Ulric Pedersen for their expert technical assistance.

\section{References}

Auer RL, Starczynski J, McElwaine S, Bertoni F, Newland AC, Fegan CD \& Cotter FE 2005 Identification of a potential role for POU2AF1 and BTG4 in the deletion of 11q23 in chronic lymphocytic leukemia. Genes,

Chromosomes and Cancer 43 1-10.

Van Buren G II, Rashid A, Yang AD, Abdalla EK, Gray MJ, Liu W, Somcio R, Fan F, Camp ER, Yao JC et al. 2007 The development and characterization of a human midgut carcinoid cell line. Clinical Cancer Research 13 4704-4712. 
Capella C, Solcia E, Sobin LH \& Arnold R 2000 Endocrine tumours of the small intestine. In Pathology and Genetics, Tumours of the digestive system, Eds SR Hamilton \& LA Aaltonen. Lyon, France: IARC Press.

Chan AO, Kim SG, Bedeir A, Issa JP, Hamilton SR \& Rashid A 2003 CpG island methylation in carcinoid and pancreatic endocrine tumors. Oncogene 22 924-934.

Choi IS, Estecio MR, Nagano Y, Kim do H, White JA, Yao JC, Issa JP \& Rashid A 2007 Hypomethylation of LINE-1 and Alu in well-differentiated neuroendocrine tumors (pancreatic endocrine tumors and carcinoid tumors). Modern Pathology 20 802-810.

Danen-van Oorschot AA, Voskamp P, Seelen MC, van Miltenburg MH, Bolk MW, Tait SW, Boesen-de Cock JG, Rohn JL, Borst J \& Noteborn MH 2004 Human death effector domain-associated factor interacts with the viral apoptosis agonist Apoptin and exerts tumorpreferential cell killing. Cell Death and Differentiation 11 564-573.

Faiss S, Pape UF, Böhmig M, Dörffel Y, Mansmann U, Golder W, Riecken EO \& Wiedenmann B 2003 Prospective, randomized, multicenter trial on the antiproliferative effect of lanreotide, interferon alfa, and their combination for therapy of metastatic neuroendocrine gastroenteropancreatic tumors - the International Lanreotide and Interferon Alfa Study Group. Journal of Clinical Oncology 21 2689-2696.

Hoeflich KP \& Ikura M 2004 Radixin: cytoskeletal adopter and signaling protein. International Journal of Biochemistry and Cell Biology 36 2131-2136.

Iafrate AJ, Feuk L, Rivera MN, Listewnik ML, Donahoe PK, Qi Y, Scherer SW \& Lee C 2004 Detection of large-scale variation in the human genome. Nature Genetics 36 949-951.

Jakobsen AM, Andersson P, Saglik G, Andersson E, Kölby L, Erickson JD, Forssell-Aronsson E, Wängberg B, Ahlman H \& Nilsson O 2001 Differential expression of vesicular monoamine transporter (VMAT) 1 and 2 in gastrointestinal endocrine tumours. Journal of Pathology 195 463-472.

Kim do H, Nagano Y, Choi IS, White JA, Yao JC \& Rashid A 2008 Allelic alterations in well-differentiated neuroendocrine tumors (carcinoid tumors) identified by genome-wide single nucleotide polymorphism analysis and comparison with pancreatic endocrine tumors. Genes, Chromosomes and Cancer 47 84-92.

Kölby L, Persson G, Franzén S \& Ahrén B 2003 Randomized clinical trial of the effect of interferon alpha on survival in patients with disseminated midgut carcinoid tumours. British Journal of Surgery 90 687-693.

Kulke MH, Freed E, Chiang DY, Philips J, Zahrieh D, Glickman JN \& Shivdasani RA 2008 High-resolution analysis of genetic alterations in small bowel carcinoid tumors reveals areas of recurrent amplification and loss. Genes, Chromosomes and Cancer 47 591-603.

Kvols LK, Moertel CG, O’Connell MJ, Schutt AJ, Rubin J \& Hahn RG 1986 Treatment of the malignant carcinoid syndrome. Evaluation of a long-acting somatostatin analogue. New England Journal of Medicine $\mathbf{3 1 5}$ 663-666.

Kwekkeboom DJ, Mueller-Brand J, Paganelli G, Anthony LB, Pauwels S, Kvols LK, O’Dorisio TM, Valkema R, Bodei L, Chinol M et al. 2005 Overview of results of peptide receptor radionuclide therapy with 3 radiolabeled somatostatin analogs. Journal of Nuclear Medicine 46 62S-66S.

Kwekkeboom DJ, de Herder WW, Kam BL, van Eijck CH, van Essen M, Kooij PP, Feelders RA, van Aken MO \& Krenning EP 2008 Treatment with the radiolabeled somatostatin analog [177 Lu-DOTA 0,Tyr3]octreotate: toxicity, efficacy, and survival. Journal of Clinical Oncology 26 2124-2130.

Kytölä S, Höög A, Nord B, Cedermark B, Frisk T, Larsson C \& Kjellman M 2001 Comparative genomic hybridization identifies loss of 18q22-qter as an early and specific event in tumorigenesis of midgut carcinoids. American Journal of Pathology 158 1803-1808.

Liu L, Broaddus RR, Yao JC, Xie S, White JA, Wu TT, Hamilton SR \& Rashid A 2005 Epigenetic alterations in neuroendocrine tumors: methylation of RAS-association domain family 1, isoform A and p16 genes are associated with metastasis. Modern Pathology 18 1632-1640.

Löllgen RM, Hessman O, Szabo E, Westin G \& Åkerström G 2001 Chromosome 18 deletions are common events in classical midgut carcinoid tumors. International Journal of Cancer 92 812-815.

Modlin IM, Lye KD \& Kidd M 2003 A 5-decade analysis of 13,715 carcinoid tumors. Cancer 97 934-959.

Novak RL \& Phillips AC 2008 Adenoviral-mediated Rybp expression promotes tumor cell-specific apoptosis. Cancer Gene Therapy 15 713-722.

Osborne DA, Zervos EE, Strosberg J, Boe BA, Malafa M, Rosemurgy AS, Yeatman TJ, Carey L, Duhaine L \& Kvols LK 2006 Improved outcome with cytoreduction versus embolization for symptomatic hepatic metastases of carcinoid and neuroendocrine tumors. Annals of Surgical Oncology 13 572-581.

Pfragner R, Wirnsberger G, Niederle B, Behmel A, Rinner I, Mandl A, Wawrina F, Luo JS, Adamiker D, Hoger H et al. 1996 Establishment of a continuous cell line from a human carcinoid of the small intestine (KRJ-I): characterization and effects of 5-azacytidine on proliferation. International Journal of Oncology 8 513-520.

Rakha EA, Green AR, Powe DG, Roylance R \& Ellis IO 2006 Chromosome 16 tumor-suppressor genes in breast cancer. Genes, Chromosomes and Cancer 45 527-535.

Rindi G, Klöppel G, Couvelard A, Komminoth P, Korner M, Lopes JM, McNicol AM, Nilsson O, Perren A, Scarpa A et al. 2007 TNM staging of midgut and hindgut (neuro) endocrine tumors: a consensus proposal including a grading system. Virchows Archiv 451 757-762.

Roche A, Girish BV, de Baere T, Baudin E, Boige V, Elias D, Lasser P, Schlumberger M \& Ducreux M 2003 
Trans-catheter arterial chemoembolization as first-line treatment for hepatic metastases from endocrine tumors. European Radiology 13 136-140.

Sablina AA, Chen W, Arroyo JD, Corral L, Hector M, Bulmer SE, DeCaprio JA \& Hahn WC 2007 The tumor suppressor PP2A Abeta regulates the RalA GTPase. Cell 129 969-982.

Sarmiento JM \& Que FG 2003 Hepatic surgery for metastases from neuroendocrine tumors. Surgical Oncology Clinics of North America 12 231-242.

Schmalhofer O, Brabletz S \& Brabletz T 2009 E-cadherin, beta-catenin, and ZEB1 in malignant progression of cancer. Cancer and Metastasis Reviews 28 151-166.

Shields JM, Rogers-Graham K \& Der CJ 2002 Loss of transgelin in breast and colon tumors and in RIE- 1 cells by Ras deregulation of gene expression through Rafindependent pathways. Journal of Biological Chemistry 277 9790-9799.

Sjögren H, Nilsson O, Behrendt M, Kölby L, Jacobsen Levin AM, Ahlman H \& Stenman G 2000 Multicolor spectral karyotype analysis of a transplantable human ileal carcinoid. International Journal of Molecular Medicine 6 629-633.

Stancu M, Wu TT, Wallace C, Houlihan PS, Hamilton SR \& Rashid A 2003 Genetic alterations in goblet cell carcinoids of the vermiform appendix and comparison with gastrointestinal carcinoid tumors. Modern Pathology 16 1189-1198.

Stridsberg M, Öberg K, Li Q, Engström U \& Lundqvist G 1995 Measurements of chromogranin A, chromogranin B (secretogranin I), chromogranin C (secretogranin II) and pancreastatin in plasma and urine from patients with carcinoid tumours and endocrine pancreatic tumours. Journal of Endocrinology 144 49-59.

Sun X, Gong Y, Talamonti MS \& Rao MS 2002 Expression of cell adhesion molecules, CD44s and E-cadherin, and microvessel density in carcinoid tumors. Modern Pathology 15 1333-1338.

Terris B, Meddeb M, Marchio A, Danglot G, Flejou JF, Belghiti J, Ruszniewski P \& Bernheim A 1998 Comparative genomic hybridization analysis of sporadic neuroendocrine tumors of the digestive system. Genes, Chromosomes and Cancer 22 50-56.

Tokunou M, Niki T, Saitoh Y, Imamura H, Sakamoto M \& Hirohashi S 2000 Altered expression of the ERM proteins in lung adenocarcinoma. Laboratory Investigation $\mathbf{8 0}$ 1643-1650.

Tönnies H, Toliat MR, Ramel C, Pape UF, Neitzel H, Berger W \& Wiedenmann B 2001 Analysis of sporadic neuroendocrine tumours of the enteropancreatic system by comparative genomic hybridisation. Gut 48 536-541.

Toyota M, Suzuki H, Sasaki Y, Maruyama R, Imai K, Shinomura Y \& Tokino T 2008 Epigenetic silencing of microRNA-34b/c and B-cell translocation gene 4 is associated with $\mathrm{CpG}$ island methylation in colorectal cancer. Cancer Research 68 4123-4132.
Walch AK, Zitzelsberger HF, Aubele MM, Mattis AE, Bauchinger M, Candidus S, Prauer HW, Werner M \& Höfler H 1998 Typical and atypical carcinoid tumors of the lung are characterized by $11 \mathrm{q}$ deletions as detected by comparative genomic hybridization. American Journal of Pathology 153 1089-1098.

Wang SS, Esplin ED, Li JL, Huang L, Gazdar A, Minna J \& Evans GA 1998 Alterations of the PPP2R1B gene in human lung and colon cancer. Science 282 284-287.

Wang GG, Yao JC, Worah S, White JA, Luna R, Wu TT, Hamilton SR \& Rashid A 2005 Comparison of genetic alterations in neuroendocrine tumors: frequent loss of chromosome 18 in ileal carcinoid tumors. Modern Pathology 18 1079-1087.

Wängberg B, Westberg G, Tylen U, Tisell L, Jansson S, Nilsson O, Johansson V, Schersten T \& Ahlman H 1996 Survival of patients with disseminated midgut carcinoid tumors after aggressive tumor reduction. World Journal of Surgery 20 892-899.

Weckström P, Hedrum A, Makridis C, Akerström G, Rastad J, Scheibenpflug L, Uhlén M, Juhlin C \& Wilander E 1996 Midgut carcinoids and solid carcinomas of the intestine: differences in endocrine markers and p53 mutations. Endocrine Pathology 7 273-279.

Westberg G, Wängberg B, Ahlman H, Bergh CH, BeckmanSuurküla M \& Caidahl K 2001 Prediction of prognosis by echocardiography in patients with midgut carcinoid syndrome. British Journal of Surgery 88 865-872.

Yang Z, Chang YJ, Miyamoto H, Ni J, Niu Y, Chen Z, Chen YL, Yao JL, di Sant'Agnese PA \& Chang C 2007 Transgelin functions as a suppressor via inhibition of ARA54-enhanced androgen receptor transactivation and prostate cancer cell growth. Molecular Endocrinology 21 343-358.

Younes N, Fulton N, Tanaka R, Wayne J, Straus FH II \& Kaplan EL 1997 The presence of K-12 ras mutations in duodenal adenocarcinomas and the absence of ras mutations in other small bowel adenocarcinomas and carcinoid tumors. Cancer 79 1804-1808.

Zhang HY, Rumilla KM, Jin L, Nakamura N, Stilling GA, Ruebel KH, Hobday TJ, Erlichman C, Erickson LA \& Lloyd RV 2006 Association of DNA methylation and epigenetic inactivation of RASSF1A and beta-catenin with metastasis in small bowel carcinoid tumors. Endocrine 30 299-306.

Zhao J, de Krijger RR, Meier D, Speel EJ, Saremaslani P, Muletta-Feurer S, Matter C, Roth J, Heitz PU \& Komminoth P 2000 Genomic alterations in well-differentiated gastrointestinal and bronchial neuroendocrine tumors (carcinoids): marked differences indicating diversity in molecular pathogenesis. American Journal of Pathology 157 1431-1438.

Zhao J, Moch H, Scheidweiler AF, Baer A, Schaffer AA, Speel EJ, Roth J, Heitz PU \& Komminoth P 2001 Genomic imbalances in the progression of endocrine pancreatic tumors. Genes, Chromosomes and Cancer 32 364-372. 